\title{
VIII CONGRESO INTERNACIONAL \\ SOCIEDAD DE ANDROLOGÍA Y GAMETOLOGIA DE CHILE \\ $X$ JORNADAS INTERNACIONALES DE VERANO EN MEDICINA REPRODUCTIVA Y BIOTECNOLOGÍA
}

\author{
Dedicadas a Prof. Dr. Jorge Correa y Prof. Dr. Renato Gatica \\ por sus trayectorias académicas.
}

\author{
Organizan \\ Universidad de La Frontera \\ Facultad de Medicina \\ Centro de Biotecnología en Reproducción (CEBIOR) \\ Sociedad de Andrología y Gametología de Chile
}

Universidad de La Frontera, Sede Pucón, 08 y 09 enero 2009

\section{Directiva}

Sociedad de Andrología y Gametología de Chile

Presidenta: Prof. Mg. Cs. Jennie Risopatrón G.

Vice-Presidente: Prof. Dr. Eduardo Bustos-Obregón

Secretaria: Prof. Mg. Cs. Mariana Deppe A.

Tesorera: Prof. Dra. Juana Villegas M.

\section{Comisión Organizadora}

Presidente: Prof. Dr. Eduardo Bustos-Obregón

Comité Organizador

Prof. Mg. Cs. Jennie Risopatrón G.

Prof. Mg. Cs. Mariana Deppe A.

Prof. Dr. Raúl Sanchez G.

Prof. Dra. Juana Villegas M.

Comité Científico y Editor

Prof. Dr. Eduardo Bustos-Obregón

Prof. Mg. Cs. Jennie Risopatrón G.

Prof. Dr. Raúl Sanchez G.

Prof. Mg. Cs. Mariana Deppe A. 
ANÁLISIS DE LA EXPRESIÓN DE RECEPTORES DE ESTRÓGENOS Y PROGESTERONA EN EL ENDOMETRIO DE OVEJAS DE LAS RAZAS ROMNEY MARSH Y ARAUCANA. (Estrogen and progesterone receptors expresion in endometrium of sheep Romney Marsh and Araucana). A. Vasconcellos ${ }^{1,2}$ M. Paredes ${ }^{2}$, J. Carrasco ${ }^{3}$, D. Nuñez3. ${ }^{1} \mathrm{CEBIOR}$ (Centro Biotecnológico de la Reproducción), Facultad de Medicina, Universidad de la Frontera, Temuco, Chile. ${ }^{2}$ Depto. Ciencias Básicas Facultad de Medicina, Universidad de La Frontera. ${ }^{3}$ Escuela de Medicina Veterinaria, Facultad de Recursos Naturales, Universidad Católica de Temuco.

El estado morfofuncional del tracto genital de las ovejas hembras es determinado por las hormonas sexuales, las que actúan a través de receptores específicos. Estos regulan la expresión de numerosas proteínas comprometidas en el desarrollo del endometrio. Posiblemente el desempeño reproductivo de diferentes razas de ovejas podría estar determinado por la expresión diferencial de los receptores. El objetivo del presente estudio fue analizar la presencia de receptores de estrógenos (RE) y progesterona $(\mathrm{RP})$ en el endometrio de ovejas prepúberes y en ciclo de las razas Romney Marsh y Araucana. La histología del endometrio se realizó en cortes de 5 $\mathrm{mm}$ teñidos con hematoxilina - eosina. La presencia de ambos receptores se detecto mediante inmunohistoquímica utilizando anticuerpos monoclonales. Además se evaluó por Dot-blot la presencia de los receptores en extractos proteicos totales obtenidos a partir de tejido endometrial. Se detectó señal inmunoreactiva contra ambos receptores en borregas y adultas ciclando de ambas razas. Se destaca inmunorreacción nuclear en células estromales y del epitelio glandular. Los RE en ovejas adultas presentan mayor expresión que en las prepuberes no encontrandose diferencias cualitativas entre ambas razas. Los RP dieron inmunoreacción positiva en ambas razas, siendo la señal levemente más fuerte en las borregas Romney Marsh, mientras que en ovejas adultas se observó mayor señal en ovejas Araucana. Concluimos que la expresión inmunohistoquímica de RE y RP es detectable en el endometrio de las ovejas variando su expresión según su etapa de desarrollo y estadio reproductivo, no pesquisándose diferencias significativas entre ambas razas.

Palabras clave: Ovejas, endometrio, receptor estrógeno, receptor progesterona, razas.
CARACTERÍSTICAS DEL MOCO CERVICAL DE MUJERES CON SÍNDROME DE OVARIO POLIQUÍSTICO (SOP) SEGÚN MICROSCOPÍA DE LUZ Y MICROSCOPÍA ELECTRÓNICA DE BARRIDO. (Cervical mucus characteristics in PCOS women under light and scanning electron microscopy) M. E. Cortés ${ }^{1,2}$ A. Zúñiga ${ }^{1}$, J. Riquelme ${ }^{1}$, P. Vigil ${ }^{1,3},{ }^{1}$ Facultad de Ciencias Biológicas, Pontificia Universidad Católica de Chile. Santiago, Chile. ${ }^{2}$ Facultad de Agronomía e Ingeniería Forestal, Pontificia Universidad Católica de Chile. Santiago, Chile. ${ }^{3}$ Fundación Médica San Cristóbal. Santiago, Chile.

*Correo-e: pvigil@bio.puc.cl

Tradicionalmente se han reconocido dos tipos de moco cervical, estrogénico y gestagénico. Éstos están constituidos por subtipos diferentes cuyas características cambian dependiendo de variaciones en los niveles hormonales y de la existencia de diversas patologías. Nuestro objetivo fue identificar las características de cristalización y ultraestructura del moco cervical de mujeres con SOP, y compararlas con las características de mujeres control. Las muestras de moco fueron tomadas de diez mujeres: cuatro del grupo control (ciclos menstruales ovulatorios) y seis con SOP (dos con ciclos ovulatorios y cuatro con ciclos anovulatorios). El moco fue caracterizado de acuerdo a cristalización y ultraestructura. El tipo de moco obtenido fue relacionado con los niveles de estradiol y progesterona presentes cuando la muestra fue tomada. En relación a las cristalizaciones del moco en las mujeres control, éstas mostraron una distribución estrogénica típica: similar a helecho $(L$, $P 2)$, rectilínea (S) o en estructura hexagonal (P6). Por otra parte, en las mujeres con SOP se encontraron cristalizaciones indefinidas de moco, así como parches de cristalización semejantes a moco estrogénico y gestagénico. En relación a la ultraestructura del moco, se encontraron diferencias entre mujeres controles y mujeres con SOP y ciclos menstruales anovulatorios, especialmente en el tipo de malla y en el diámetro promedio de los poros de la malla de moco. Los resultados anteriormente expuestos muestran que las características de cristalización y ultraestructura del moco cervical en las mujeres con SOP son diferentes a las de mujeres control. Estas diferencias dependerían de sus niveles de estrógenos y progesterona.

Palabras clave: Moco cervical, mujeres SOP, microscopía óptica y de barrido. 
CARACTERIZACIÓN DE LAS PROTEINAS EN LA SEGUNDA FRACCIÓN DEL PLASMA SEMINAL CANINO. (Protein characterization in the second fraction of canine seminal plasma). J. Villegas ${ }^{1,2}$ O. Rosales ${ }^{1}$, J. Risopatrón ${ }^{1,3}$, R. Sánchez ${ }^{1,4}{ }^{1}$ Centro de Biotecnología en Reproducción, ${ }^{2}$ Depto. Medicina Interna, ${ }^{3}$ Depto. Ciencias Básicas, ${ }^{4}$ Depto. Ciencias Preclínicas, Facultad de Medicina, Universidad de La Frontera, Temuco, Chile.

jvillega@ufro.cl

En diversas especies se ha demostrado la importancia de las proteínas del plasma seminal para la fecundación. Dichas proteínas se unen a la membrana espermática durante la eyaculación y previenen la capacitación hasta el momento en que el espermatozoide encuentra al ovocito. Estas propiedades fundamentan el uso de plasma seminal en procedimientos de crioconservación de semen. El objetivo de este trabajo fue caracterizar las proteínas presentes en la segunda fracción del plasma seminal canino.Se obtuvieron muestras de segunda fracción de 40 eyaculados de 2 perros. Alícuotas de pool de plasma seminal se filtraron a través de membranas de MWCO 30 y $10 \mathrm{kDa}$ por centrifugación a 14.000rpm durante $1 \mathrm{~h}$. y se analizaron por electroforesis SDS-PAGE en geles 15\%.La concentración de proteínas del pool de segunda fracción fue de $9,35 \mathrm{mg} / \mathrm{ml}$. En la electroforesis se observan 11 bandas de 75, 66, 28, 24, 22, 18,16.5, 9.5, 5.5, 4kDa. En el filtrado de proteínas menores a $30 \mathrm{kDa}$ se observan las bandas de $75,66,28,16.5$ y $9.5 \mathrm{kDa}$. Las 2 bandas de 16.5 y $9.5 \mathrm{kDa}$ coinciden por tamaño molecular y concentración con las subunidades de proteína específica prostática canina, proteína de $29 \mathrm{kDa}$ que constituye el $30 \%$ de las proteínas del plasma seminal.

FONDECYT 1070594

\section{DESARROLLO EMBRIONARIO IN VITRO DE OVOCITOS INMADUROS DE BOVINO VITRIFICADOS. (In vitro embrionic development of bovine immature vitrified oocyte). M Schulz ${ }^{1}$, J Risopatrón ${ }^{1,2}, \mathrm{P} \mathrm{Bravo}^{3}, \mathrm{R}$ Sánchez ${ }^{1}{ }^{1}$ Centro de Biotecnología en Reproducción, ${ }^{2}$ Dpto Cs. Básicas, Facultad de Medicina, Universidad de La Frontera, Temuco, Chile.}

La crioconservación de embriones de bovino es un procedimiento rutinario en ganadería e industria de transferencia embrionaria. Distintas técnicas de vitrificación han surgido para obtener mayores tasas de sobrevivencia a través de una reducción del volumen de solución de vitrificación y utilizando distintos tipos de crioprotectores. El objetivo de este trabajo fue evaluar el desarrollo embrionario post fecundación in vitro de ovocitos de bovino vitrificados con DPBS+sucrosa $0.3 \mathrm{M}+$ Etilenglicol $20 \%$ y DMSO, en pajuelas cut standar straw (CSS).Los ovocitos se obtuvieron mediante punción de ovarios de vacas faenadas (Frigorífico Temuco), y fueron divididos en dos grupos: A) ovocitos maduros (24hr) y luego vitrificados, y B) ovocitos inmaduros vitrificados inmediatamente. Luego de la desvitrificación los ovocitos del grupo A fueron fecundados. En el caso del grupo B, luego de desvitrificados, los ovocitos fueron madurados y posteriormente fecundados. En ambos grupos se usaron espermatozoides de semen congelado en pajuelas. Cumplidas 48 horas de incubación los cigotos fueron observados en microscopio invertido (20X) y la presencia de división celular de 2 ó más células fueron consideradas como signo de desarrollo embrionario. En el grupo A se obtuvo un 20,0 $\pm 0,8 \%$ de desarrollo embrionario y en el grupo $B$ un $22,5 \pm 1,3 \%(p<0.05)$. Con estos resultados preliminares, se puede concluir que la vitrificación de ovocitos inmaduros presenta mayor desarrollo embrionario, ya que al presentar el cúmulo compacto conserva mejor la integridad celular y metodológicamente es más fácil depositarlos en las pajuelas CCS.

Financiamiento: DI07-0104 Universidad de La Frontera.

Palabras clave: immature oocyte, vitrification, embryo, bovine.

\section{DISTRIBUCIÓN DE AMPK EN EPITELIO SEMINÍFERO DE RATAS NORMALES Y DIABÉ- \\ TICAS. (AMPK distribution in normal and diabetic rat seminieirous epithelium). A. Ramírez'; R. Gatica²; I. Concha²; A. Yañez ; J. Slebe ${ }^{2}$. Intituto de Ciencia Ani- mal, Unidad de Reproducción, Facultad de Ciencias Veterinarias ${ }^{1}$. Instituto de Bioquímica, Facultad de Cien- cias $^{2}$. Universidad Austral de Chile.}

La Diabetes Mellitus (DM) tipo I es un síndrome metabólico caracterizado por una hiperglicemia, destrucción de las células b-pancreáticas y una disminución en la producción y secreción de insulina. Asimismo se ha determinado que alrededor del $90 \%$ de los pacientes diabéticos presentan alteraciones en la función sexual, destacando la pérdida de libido, la impo- 
tencia y la infertilidad. En el caso de la infertilidad, la disfunción testicular está altamente asociada con la progresión de la diabetes (hiperglicemia). La función de AMPK ha demostrado ser importante en el metabolismo energético de diversos tejidos participando en el control celular anabólico y catabólico. El objetivo de este estudio fue determinar la distribución y estado de activación de AMPK en testículo de ratas normales y diabéticas. Para ello, se realizó análisis inmuno-histoquímico en epitelios seminíferos de ratas normoglicemicas y ratas hiperglicemicas. La condición diabética fue inducida mediante administración de estreptozotocina. Los resultados indican que AMPK esta presente en células de Leydig, Sertoli, espermatogonias y espermatidas. En condiciones de DM tipo I AMPK mostró un aumentó en la expresión en todos los tipos celulares del epitelio seminífero. Los resultados sugieren que diabetes induce cambios en la expresión y localización de AMPK con bajos niveles de fosforilación de AMPK. Entender el papel de AMPK en el control de la función testicular normal y patológica podría tener importantes implicancias en el manejo farmacológico de la infertilidad asociada a la hiperglicemia.

Palabras clave: Distribución AMPK, epitelio seminífero, rata diabética y normal.

\section{EFECTO DE LA CURVA DE ENFRIAMIENTO A $5^{\circ} \mathrm{C}$ SOBRE LA CALIDAD DE SEMEN OVINO} CRIOPRESERVADO.(Effect of cooling rate on cryopreservated ram semen quality). M. Muñoz, K. Inostroza, S. Bravo, N. Sepúlveda. Facultad de Cs. Agropecuarias y Forestales, Centro de Biotecnologia en Reproducción, Universidad de la Frontera.

Es conocido que en el proceso de criopreservación los espermatozoides sufren un daño importante que limita su capacidad fecundante posterior. El objetivo de este trabajo fue comparar diferentes velocidades de enfriamiento para evaluar calidad seminal postdescongelación. Semen de carnero de raza Romney Marsh fue colectado mediante una vagina artificial $\left(a 37^{\circ} \mathrm{C}\right)$, se evaluó su motilidad y recuento de espermatozoides, para la posteriormente diluir con una solución de fructosa-tris-yema de huevo - glicerol y en pajuelas de $0,25 \mathrm{ml}$ (100 $\times 10^{6}$ espermatozoides) semen. Los tratamientos realizados consistieron en la disminución de la temperatura de enfriamiento a una tasa de 0,$2 ; 0,5 ; 1$ y $3^{\circ} \mathrm{C} / \mathrm{min}$. La evaluación de dichos tratamientos, consistió en comparar motilidad, viabilidad, capacitación espermática y reacción acrosomal de las muestras en estado fresco, luego del proceso de enfriamiento y al momento del descongelado. Los resultados obtenidos indican que a una velocidad de enfriado de $0,2^{\circ} \mathrm{C} / \mathrm{min}$ se observan los mayores porcentajes de motilidad y de espermatozoides vivos, existiendo diferencia significativa $(p \leq 0,05)$ con respecto a los resultados obtenidos en las otras velocidades evaluadas. Asimismo, ocurre con los porcentajes de capacitación espermática y reacción acrosomal, donde se obtiene una relación directamente proporcional, ya que a mayor velocidad de enfriado, mayores son los resultados de espermatozoides capacitados y reaccionados, existiendo diferencia significativa $(p \leq 0,05)$ entre los grupos evaluados. En conclusión, la velocidad de enfriado que presenta mejores resultados de motilidad y viabilidad espermática corresponde a $0,2^{\circ} \mathrm{C} / \mathrm{min}$.

Palabras clave: cooling rate,
cryopreservation, ram.

\section{EFECTOS FISIOLÓGICOS DE GABA, ESTRADIOL Y PROGESTERONA EN LA REAC- CIÓN ACROSÓMICA DEL ESPERMATOZOIDE}

(Physiological effects of GABA, estradiol and progesterone upon sperm acrosomic reaction). $\mathrm{R}$. Orellana $^{1}$, V. Barrientos ${ }^{1}$, A. Godoy ${ }^{1}$, P. Vigil ${ }^{1,2},{ }^{*}{ }^{1} \mathrm{Fa}-$ cultad de Ciencias Biológicas, Pontificia Universidad Católica de Chile. Santiago, Chile. ${ }^{2}$ Fundación Médica San Cristóbal. Santiago, Chile.

*correo-e: pvigil@bio.puc.cl

El ácido g-aminobutírico (GABA) ha sido descrito como un inductor "dosis-dependiente» de la reacción acrosómica (RA) del espermatozoide. La progesterona (P4), por su parte, promueve la RA y su acción es antagonizada por el 17b-estradiol (E2). El objetivo de este trabajo es confirmar la «acción estrogénica» en los efectos de GABA, descrita en el sistema nervioso; además, determinar el efecto de la adición conjunta de GABA y $\mathrm{P} 4$, en el \% RA. A partir de espermatozoides humanos capacitados durante $2 \mathrm{~h}$ en medio BWW (4 \% de albúmina de suero bovino, BSA), se realizaron las siguientes incubaciones: BWW + BSA $4 \%$ (Control), P4, E2, GABA, P4 + GABA y E2 + GABA a tiempos distintos. Mediante tinción Lectina Pisum sativum se determinó el \% RA. La incubación E2 + GABA mostró valores menores $(17 \pm 1,17)$ de $\%$ RA respecto a GABA $(33 \pm 1,16)$. La administración conjunta de P4 + GABA $(34 \pm 2,8)$ no difiere significativamente respecto a la administración individual de cada hormona (GABA: $33 \pm 1,16$; P4: $33 \pm 2,04$ ). Nuestros resultados confirman la acción moduladora de E2 en los efectos de GABA, además sugieren que la administración conjun- 
ta de P4 y GABA tiene efectos similares respecto al suministro individual de cada hormona, descartando la existencia de efectos sinérgicos entre ambas. Concluimos que la RA estaría regulada por P4, GABA y E2. Las oscilaciones periódicas de dichas hormonas en el tracto femenino podrían determinar la ocurrencia oportuna de la RA y del proceso de fertilización.

Palabras clave: GABA, estradiol, progesterona, reacción acrosómica.

\begin{abstract}
EVALUACION DE IBUPROFENO Y MELATONINA COMO AGENTES PROTECTORES A NIVEL TESTICULAR EN RATONES SOMETIDOS A HIPOXIA HIPOBARICA CONTINUA E INTERMITEN-

TE. (Ibuprofen and melatonin evaluation as protective agents in mouse testis submitted to hypobaric continuos or intermittent hypoxia). A. Vargas; E. BustosObregón. Laboratorio de Biología de la Reproducción, ICBM, Facultad de Medicina, Universidad de Chile. Santiago, Chile. aavargas@med.uchile.cl
\end{abstract}

La menor concentración de oxígeno disponible repercute en diferentes órganos, incluyendo el sistema reproductor masculino. Hipoxia hipobárica continua $(\mathrm{HH})$ e intermitente $(\mathrm{HI})$ producen neoangiogénesis (aumentando la temperatura intraescrotal) y elevan la producción de especies reactivas de oxigeno en testículo. Se evaluó daño testícular post hipoxia en tres grupos de ratones: controles ( $500 \mathrm{msnm}$ ), $\mathrm{HI}$ (4 días en hipoxia y 4 en normoxia) y HH en cámara hipobárica (4200 msnm ) durante 33,2 días. Se administró el antiinflamatorio no esteroidal Ibuprofeno (antiangiogénico) y la hormona Melatonina (antioxidante) para evaluar el efecto protector testicular. Se determinó; parámetros hematológicos, masa testicular y de cauda epididimaria, recuento, morfología y peroxidación lipídica espermática, análisis morfométrico e histopatológico de Túbulos Seminíferos, grado de descompactación de la cromatina, fragmentación y estabilidad del ADN espermático, y cuantificación inmunohistoquímica de HIF-1 a. HH e HI aumentó significativamente el hematocrito y los reticulocitos, la fragmentación, descompactacion e inestabilidad del ADN, la expresión de HIF-1 a, la teratozoospermia, las alteraciones histopatológicas en túbulos seminíferos y disminuyó el recuento espermático. En $\mathrm{HI}$ el grado de peroxidación lipídica aumento significativamente y disminuyó el peso de cauda epididimaria. Melatonina redujo significativamente la inestabilidad del ADN y la peroxidación lipídica en $\mathrm{HH}$ e $\mathrm{HI}$ y redujo la tetatozoospermia solo en $\mathrm{HH}$.lbuprofeno redujo significativamente las alteraciones histopatológicas de túbulos seminíferos en $\mathrm{HI}$ y aumentó significativamente el recuento espermático en $\mathrm{HH}$. Redujo la descompactación, fragmentación, inestabilidad del ADN y la expresión de HIF1 a en $\mathrm{HH}$ e HI. Conclusión: $\mathrm{HH}$ e $\mathrm{HI}$ producen daño testicular disminuyendo la fertilidad. Ibuprofeno ejerció mayor protección que melatonina disminuyendo las alteraciones de los parámetros analizados.

Palabras clave: Hipoxia, Ibuprofeno, melatonina, HIF-1 a.

\section{FRACCIONES PROTEICAS DEL FLUIDO SEMINAL COMO MEDIO CRIOPROTECTOR DE ESPERMATOZOIDES CANINOS. (Cryoprotective use} of seminal fluid proteinic factors in canine sperm).M. Muñoz, F. Treulen, N. Sepúlveda, R. Sánchez, J. Risopatrón. Centro de Biotecnología en Reproducción, Facultad de Medicina, Universidad de La Frontera, Temuco-Chile.

Los procedimientos de criopreservación espermática inducen efectos deletéreos sobre la estructura celular, afectando la longevidad, movilidad y capacidad fecundante. Detectar estos daños es relevante, lo que insta a optimizar los criprotectores evaluando biomoléculas fisiológicas crioprotectoras, que protejan la funcionalidad celular, entre las cuales se destacan las Proteínas del Fluido Seminal (PFS). El objetivo de este trabajo fue evaluar el efecto crioprotector de PFS de diferentes peso molecular, en la congelación de espermatozoides caninos. Se realizaron 5 grupos experimentales. Como grupo Control (C) se utilizó el método comercial Uppsala Equex-2 System 's, y en los grupos experimentales se reemplazo el Equex por diferentes fracciones de PFS: (I:<10KD; II:<30KDa; III:<100KDa y el IV adicionando FS $3^{\circ}$ fracción). Se evaluó Viabilidad (PI), Integridad de la Membrana (SYBR-14/PI), translocación de fosfatidilserina (Anexina- $V$ ), integridad membrana acrosomal (PSA/FITC-PI), integridad membrana mitocondrial (JC-1), por citometría de flujo. No se observan diferencias entre los grupos experimentales y con el control. Por los resultados se concluye que las PFS poseen un efecto crioprotector, similar a los reportados con el método comercial, esto permite sugerir su utilización como una alternativa fisiológica en la criopreservación de espermatozoides caninos.

\section{Palabras clave: Congelación, espermazoides, canino, fluido seminal.}

Financiamiento: FONDECYT №1070594 (RS) 
FRAGMENTACIÓN DE ADN EN ESPERMATOZOIDES VITRIFICADOS TRUCHAARCOIRIS (Oncorhynchus mykiss) Y SU RELACIÓN CON LA FECUNDACIÓN. (Fragmentation of DNA in rainbow trout (Oncorhynchus mykiss) vitrified spermatozoa and its relationship with fertilization.) $\mathrm{O}$. Merino'; E. Figueroa'; I. Valdebenito'; J. Risopatrón 1,3

${ }^{1}$ Centro de Biotecnología en Reproducción, Universidad de La Frontera, ${ }^{2}$ Escuela de Acuicultura, Universidad Católica de Temuco. ${ }^{3}$ Depto. Cs. Básicas, Facultad de Medicina, Universidad de La FronteraChile.

La criopreservación de semen de peces aún no es ampliamente utilizada debido a que después de descongelar la viabilidad y la fecundidad de los espermatozoides se reducen drásticamente como consecuencia del daño celular que surge por el proceso de congelación-descongelación, en espermatozoides de Trucha arcoiris, Corvina Atlántico y Lubina han observado una significativa fragmentación del ADN. Sin embargo en la crioconservación por vitrificación no existen antecedentes. El objetivo del presente trabajo fue evaluar el efecto de la vitrificación sobre la fragmentación del ADN y su relación con fecundidad. Los espermatozoides fueron vitrificados en medios: $A$. Cortland suplementado con sucrosa y plasma seminal y B. Cortland (control). Alícuotas de $20 \mu \mathrm{L}$ de suspensión espermática fueron vitrificadas directamente en N2L. La desvitrificación se realizó Cortland $\left(10^{\circ} \mathrm{C}\right)$. Se evaluó post-vitrificación espermática: fragmentación del ADN por la técnica de "TUNEL" con el kit comercial In situ cell death detection fluorescein (ROCHE), por citometría de flujo. La fecundación se determino por presencia de tubo neural en embriones incubados por 7 días a $9^{\circ} \mathrm{C}$.Los resultados preliminares con medio A indican porcentaje de fragmentación de ADN mayor al control $(21,13 \pm 12,75 \%$ v/s $9 \pm 6,3 \%)$. Con respecto al porcentaje de fecundación, fue $32 \pm 12,17 \%$ v/s $4 \pm 2 \%$, respectivamente. Al comparar los resultados previamente obtenidos después de la vitrificación con respecto a la integridad de membrana y potencial mitocondrial, el daño en el ADN es menor. Es importante continuar la investigación para obtener optimizar la técnica de vitrificación.

Palabras clave: Criopreservación, Fragmentación ADN, Vitrificación, Fecundación.

Financiamiento: Proyecto FONDEF IDO6I1020
INMUNODETECCIÓN DE LA PROTEÍNA DEL PRION (PRPC) EN GAMETOS Y DURANTE EL DESARROLLO EMBRIONARIO BOVINO. (Immunodetection of the prion protein (PrPC) in gametes and during bovine embryonic development). K. Gudenschwager ${ }^{1}$; O. Peralta ${ }^{1,2}$; W. Eyestone ${ }^{2} ;{ }^{1} \mathrm{Fa}-$ cultad de Ciencias Veterinarias, Universidad Austral de Chile, Valdivia, Chile; ${ }^{2}$ Virginia-Maryland Regional College of Veterinary Medicine, Virginia Tech, Blacksburg, USA. oscarperalta@uach.cl

La proteína celular del prion (PrPC) es una glicoproteína que se encuentra normalmente adherida a la membrana plasmática por un anclaje de glicofosfoinositol (GFI). Bajo ciertas condiciones, PrPC puede adoptar una configuración patogénica conocida como PrPSc, la cual es considerada como agente infeccioso de las encefalopatías espongiformes transmisibles (EETs). A pesar de que la función de PrPC no ha sido esclarecida, existen reportes que sugieren su participación en división y diferenciación celular. El objetivo del presente trabajo fue determinar la expresión de PrPC en gametos y en distintos estados de desarrollo embrionario en el bovino. Embriones preimplantacionales al día 4 y 8 fueron producidos desde ovocitos obtenidos de matadero, utilizando fecundación in vitro (FIV). La obtención de embriones post-implantación se realizo través de lavado intrauterino no quirúrgico (días 14 y 18) e histerectomía (días 27,32 y 39). Para immunofluorescencia se utilizo anticuerpos SAF-32 y Alexa-fluor 488 y 594. La técnica de western blot fue realizada utilizando SAF-32 e IgG asociada a fluorescencia y cuantificada por sistema infrarrojo. PrPC fue inmunodetectada en el segmento ecuatorial del espermatozoide, citoplasma del ovocito y asociada a blastómeras (día 4), macizo celular interno (día 8) y trofoblasto (días 8 y 18). El análisis de western blot mostro bandas de 31 y $29 \mathrm{kDa}$ a los días 27,32 y 39 , las cuales no evidenciaron intensidades distintas entre días $(1 ; 1,20$ y 1,45 relativo a día $27 ; \mathrm{P}<0,05)$. La expresión de PrPC en estados tempranos de desarrollo sugiere un rol en el proceso de embriogénesis.

Palabras clave: Inmunodetección, Prion (PRPc), desarrollo embrionario, bovino.

LA INHIBICIÓN DE LA PROTEÍNA QUINASA C
(PKC) PREVIENE LA MUERTE DE
ESPERMATOCITOS EN CULTIVO. (Inhibition of
protein kinase C (PKC) prevents spermatocytic death
in cultures). X. Bustamante-Marin ${ }^{1}$; J. Gutierrez; A. 
Quest. R. Moreno ${ }^{1}$ Facultad de Ciencias Biológicas, Pontificia Universidad Católica de Chile. Facultad de Medicina Universidad de Chile

En la espermatogenesis, la apoptosis es necesaria para mantener la razón entre el número de células de Sertoli y germinales. Las señales que regulan la apoptosis no se han dilucidado completamente. Se ha sugerido que es ocasionada por un aumento en la producción de especies reactivas de oxigeno (ROS), activación de caspazas y la regulación de los niveles de receptores como FAS y c-kit. En células somáticas se ha asociado la participación de miembros de la familia de PKC con el aumento de la producción de ROS. Nuestro objetivo fue determinar si la inhibición de PKC previene la muerte de espermatocitos en cultivo. Los espermatocitos se cultivaron en medio sin sustrato o suplemementado con glucosa o lactato en presencia - ausencia de los inhibidores de PKC Bisindolylmaleimida, Rottlerin y G06976. Para evaluar el efecto de la activación de PKC los espermatocitos se cultivaron en presencia de forbol-metil-acetato (PMA). La muerte se evaluó mediante la incorporación de azul de tripan y la apoptosis mediante incorporación de Anexina V. La producción de ROS y cambios en los niveles de FAS y c-kit se evaluaron mediante citometría de flujo. Nuestros resultados muestran que la inhibición de PKC previene la muerte, la generación de ROS y la disminución de c-kit en espermatocitos en cultivo.

Palabras clave: Proteína kinasa C, esparmatocitos, cultivo, muerte celular.

Fondecyt 1070360.

NIVELES INTRACELULARES DE ESPECIES
REACTIVAS DE OXÍGENO (ROS) EN
ESPERMATOZOIDES DE CANINO REFRIGERADOS A $4{ }^{\circ} \mathrm{C}$. (Intracellular reactive oxygen species levels in canine spermatozoa refrigerated at $4{ }^{\circ} \mathrm{C}$ ). Barrera, M. ${ }^{1}$,Villegas, J. ${ }^{1,2}$,Sánchez R. ${ }^{1,3}$, Risopatrón, J. ${ }^{1,4}$. Centro de Biotecnología en Reproducción; ${ }^{2}$ Depto. Medicina Interna; ${ }^{3}$ Depto. Ciencias Preclínicas, 4Depto. Ciencias Básicas, Facultad de Medicina, Universidad de La Frontera.

mbarrera@ufro.cl

La movilidad de espermatozoides de canino se ve disminuida luego de la refrigeración por más de 10 días. La criopreservación expone al espermatozoide a condiciones de estrés que generan ROS, los que causarían una rápida disminución de la concentración del ATP intracelular y pérdida de la movilidad espermática. El objetivo fue evaluar niveles intracitoplasmáticos de ROS e integridad de membrana en espermatozoides caninos preservados a $4{ }^{\circ} \mathrm{C}$ hasta 240 horas. En espermatozoides suspendidos en medio tris-yema de huevo se evaluó ROS intracitoplasmático (DHE/SYTOX green) y la integridad de membrana (SYBR-14/PI), antes y después de 2,120 y $240 \mathrm{hrs}$. de refrigeración a $4{ }^{\circ} \mathrm{C}$. Se observó un aumento aunque no significativo de la concentración intracitoplasmática de ROS a las 240 horas de refrigeración respecto al control. La integridad de membrana disminuyó a las 120 y 240 horas de refrigeración respecto al control. Estos cambios tampoco fueron significativos.La disminución de la movilidad durante la refrigeración de los espermatozoides caninos no está asociada a pérdida de la integridad de la membrana ni al aumento del nivel intracitoplasmático de ROS. La pérdida de movilidad podría atribuirse a disminución de factores que aportan requerimientos energéticos a estas células.

\section{FONDECYT Nº1070594}

\section{VITRIFICACIÓN ESPERMÁTICA EN "NEOMACHOS" DE TRUCHA ARCOIRIS (Oncorhynchus mykiss): EFECTO EN LA FRAGMENTACIÓN DEL DNA Y CAPACIDAD FECUNDANTE DE LOS ESPERMATOZOIDES POST-VITRIFICADOS. (Vitrification sperm in "neomachos" rainbow trout (Oncorhynchus mykiss): effect on the fragmentation of DNA fertilization and ability of sperm post-vitrified). E. Figueroa ${ }^{1}$; O. Meri- no ${ }^{1}$; I. Valdebenito ${ }^{2}$; J. Risopatrón ${ }^{1,3}{ }^{1}$ Centro de Biotecnología en Reproducción, Universidad de La Frontera, ${ }^{2}$ Escuela de Acuicultura, Universidad Católi- ca de Temuco. ${ }^{3}$ Depto. Cs. Básicas, Facultad de Medi- cina, Universidad de La Frontera.}

Actualmente se ha demostrado que la vitrificación preserva la función y motilidad espermática en mamíferos en forma altamente efectiva, procedimiento simple y de menor costo que la congelación tradicional. Sin embargo en peces no ha sido evaluada. El objetivo de este trabajo fue vitrificar espermatozoides en "neomachos" de trucha arcoiris (Oncorhynchus mykiss) y evaluar la fragmentación del DNA y la capacidad fecundante post-vitrificación. Los espermatozoides fueron vitrificados en medios: A. 
Cortland (control) y B. Cortland suplementado con sucrosa $(0,13 \mathrm{M})$, dimetil sulfóxido (DMSO) y plasma seminal (PS). Alícuotas de $30 \mu \mathrm{L}$ de suspensión espermática fueron vitrificadas dejándolas caer rápida y directamente en N2L. La desvitrificación se realizó en medio Cortland $\left(10^{\circ} \mathrm{C}\right)$. Se evaluó postdesvitrificación espermática: fragmentación del DNA (TUNEL) por citometría de flujo (FACSCalibur, Becton Dickinson). La fecundación se evaluó la presencia de tubo neural en embriones de 7 días de desarrollo a $9^{\circ} \mathrm{C}$. Los espermatozoides vitrificados con medio $\mathrm{B}$, presentaron menor porcentaje de fragmentación del DNA $(11.3 \pm 3,4 \%)$ sin diferencias estadísticamente significativas con respecto al control $(18.1 \pm 6,1 \%)$. Los resultados observados en los porcentajes de fecundación $(30,5 \pm 3 \%)$ con medio $B$, presentaron diferencias estadísticamente significativas con el control $(9,6 \pm 7 \%)$. Estos resultados preliminares sugieren que la vitrificación de espermatozoides en "neomachos" de trucha arcoiris con sucrosa, DMSO y plasma seminal, disminuyen el criodaño, pero se debe continuar con la investigación para optimizar resultados.

Palabras clave: Vitrificación, Espermatozoide, fragmentación del DNA, fecundación

Financiamiento Proyecto: FONDEF IDO6I1020 